\title{
OPTICAL WAVEGUIDE SENSORS
}

\section{J FLUITMAN and Th POPMA}

Twente University of Technology, POB 217,7500AE Enschede (The Netherlands)

(Recelved April 1986, accepted June 26, 1986)

\begin{abstract}
An overview of the field of optical waveguide sensors is presented Some emphasis is laid on the development of a single scheme under which the diversity of sensor principles can be arranged Furst three types of sensors are distinguished intrinsic, extrinsic and active Next, two steps are distınguished in the sensing process the physical alteration of the waveguide (via geometrical and/or materials effects) and the effect thereof on the guided light (changing phase and/or amplitude of the light modes) The diversity and common features of the field of optical waveguide sensors are demonstrated with the help of many examples
\end{abstract}

\section{Introduction}

Optical sensors generally consist of a source of light, a number of opt1cal components to lead the light in the proper shape to some modulating agent and finally a detector, possibly again preceded by some optical components This configuration is immediately derived from our own method of vision With our eyes we detect shapes, colours, movement, etc in a properly lluminated scene Optical components are necessary if we want to detect more detalls or to select the aspects we are interested in The optical microscope is a classical example of a set of such components with a high degree of sophistication Although human vision can serve as a starting point for our treatment, the subjects of our considerations generally are instruments with added electronics Light sources and detectors are electro-optic components that can be controlled, while signals and information can be processed to lead to a convenient output

The central part of the optical sensor system is the modulator, this survey will focus on that part, so we will concentrate on how light can be modulated or influenced at all and how this can be exploited in a sensing instrument A practical problem with optical sensors has been how to shape and guide the light to this modulator The use of lenses, diaphragms, mirrors, prisms, etc is inevitable in most cases using a classical light source This can lead to a complicated and expensive construction in a workung instrument 
The invention of the laser as a source of well-directed light has led to great improvements and at the same time has given rise to a number of new principles To mention one, the pencll of light can be used to scan profiles in order to learn their precise shape Of course, the high degree of coherence of laser light has led to a large number of new developments

The invention of the optical waveguide, however, is of decisive importance, as it is a means for point-to-point transportation of optical energy and of the information that may be carried by it This led to a breakthrough in optical sensing, since it is no longer necessary to have the light source and the detector close to the modulator This means that places, inaccessible up to now, are within reach of optical sensing methods This advantage was recognized long before the optical fibre could be produced with the quality necessary for long distance communication In the 1960s, for instance, the determination of mechanical parameters like position or vibration could be detected with the help of fibre bundles, in which part of the fibres carried the light to some object, while the others caught the light reflected from it Also, researchers in the field of biomedical engineering were soon attracted to the possibilities of the tiny probelike fibre, for obvious reasons

With the possible integration of 'all-optic' sensing and communication of the acquired information, the advantages of the latter are shared by the furst These advantages are immunity for EMI (ignition pulses, lightning strokes, etc ), no crosstalk, no necessity for metal parts, no chance of sparks, etc These advantages can be exploited in sensors operating in harsh environments (power stations), in inflammable or explosive environments (petrochemical industry, drilling sites), in biomedical applications and so on (the list can easily be extended, see, e g, Main [1])

Just as in the case of the laser, the optical waveguide has led to a number of new sensor principles So, with respect to optical sensing, the role of the waveguide need not be limited to the mere guidance of light to and from the modulator, but the waveguide may be considered as an optical component that can itself be modulated Even very small influences can be made detectable by colling a long length of fibre in the modulator area This led to substantial research activities, which began during the 1970s and gave the field of optical waveguide sensors a certain identity

Considered as optical components, optical fibres are not the only waveguides of interest Although they are the most accessible for sensor applications at the moment, strip waveguides from the field of integrated optics are at least as attractive A difficult point is that such integrated optical components should be inserted in an optical communication line, which makes the avalability of reliable fibre-to-strip coupling a necessity (otherwise the inherent advantages of optical waveguide sensors are lost) On the other hand, the avallability of planar technology and the accessibility of this technology for matenals, forms and principles to exploit all challenges of physics will gradually emphasize the role of integrated optical devices

To reiterate, the optical waveguide can serve two purposes, but not both at the same time The waveguide can be used as a component to guide 
light in suitable form to and from the modulator part, or the waveguide can be used as the modulator part itself These viewpoints are rather different and often originate from different groups of people those from the sensor field who discovered the waveguide (the present authors belong to this group) and those from the waveguide field who discovered the sensors This may lead to a discussion on how an optical waveguide sensor should be defined To embrase both viewpoints, we define the optical waveguide sensor as a sensor with a configuration as mentioned in the first lines of this Introduction, while an optical waveguide is an essential part of it for whatever reason

Because of the divergent viewpoints mentioned above, we have made it our first object to develop a single scheme under which the diversity of sensor principles could be classified in good order It has not been our intention to give a complete overview of the literature Many surveys have recently been produced [2 - 5], which can serve this purpose With the help of these surveys, their large number of citations and the literature mentioned here, an interested reader will easily find more information

Our second intention is to give the reader who is not famuliar with the subject a possibility to appreciate the field of optical waveguide sensors Therefore we will give a short treatise on the principles of optical waveguides in the next Section Then we analyse the principles of optical waveguide sensing in order to classify the field After that we will give a survey of charactenstic developments

\section{Waveguide optics}

In order to understand the operation of a large number of optical waveguide sensors, it it necessary to have an understanding of light propagation through those guides As the word 'waveguide' indicates, light in such a device is more or less forced to follow a prescribed trajectory In the furst instance an optical fibre can be seen as a flexible tube constructed in such a way that light rays are kept inside as a consequence of internal reflections and are forced to propagate in the axial direction As long as the fibres are relatively thick (in comparison with the wavelength of the light) this description in terms of geometrical optics works well, but if the fibres are small in diameter, the wave character of light will dominate the description and this leads to rather complex expressions and notions Therefore we wll first focus our attention on planar optical waveguides because these are easier to treat, whlle their essential properties are transferable to the more complex case of fibres

A planar optical waveguide is sketched in Fig 1 . In fact we consider a slab which extends to infinity in both the $y$-and $z$-durections, but we confine ourselves to light waves propagating in the $z$-direction An obvious way to keep light within the slab is to use transparent materials with reiractive indices $n_{2}<n_{1} \quad$ A ray of light inside the slab that hits the boundary at an 


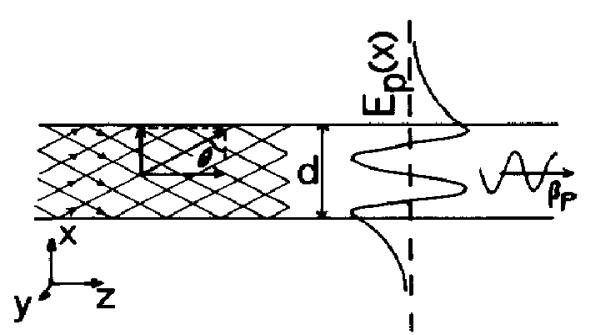

Fig 1 Symmetric planar optical waveguide consists of layer of index $n_{1}$ sandwiched between material with index $n_{2}<n_{1}$ Guided light modes propagate with wavenumber $\beta_{p}$ and have a standing wave structure fitting in the $x$-durection

angle $\theta$ larger than the critical angle $\theta_{c}\left(\sin \theta_{c}=n_{2} / n_{1}\right)$, will be totally reflected at both sides and thus be guided in the axial direction

This suggests that any ray having $\theta>\theta_{c}$ could be guided through the slab However, the notion of a wavefront running in the upward sense in one part of the guide and downward in the next is incorrect in a slab with a thickness of the order of the wavelength of the light In fact both orientations run through each other in all parts of the slab and this gives rise to interference effects, which are constructive for only a restricted set of $\theta$-values

This set determines the modes of guided light waves They have the structure of a standing wave that 'fits' in the $x$-direction and of a running wave in the durection of propagation $z$ according to

$E=E_{p}(x) \exp 1\left(\omega t-\beta_{p} z\right)$

Here $E$ stands for a component of the electric or magnetic field vector, $\omega$ is the frequency, $\beta_{p}$ the wavenumber of the running part, while $p$ is a mode number As can be seen from Fig 1 , the standing wave part $E_{p}(x)$ is not strictly confined to the slab (this would be the case if the slab was bound by perfect conductors), but extends somewhat into the claddıng region (the slab region is called the core) This feature follows from a more rigorous treatment of the problem, which is equivalent to solving Maxwell's equations and selecting those solutions that fit at the interfaces

Note that from $\pi / 2>\theta>\theta_{\mathrm{c}}$ for guded waves, it follows that

$n_{2} \omega / c<\beta_{p}<n_{1} \omega / c$

For $\theta<\theta_{c}, l e, \beta_{p}<n_{2} \omega / c$, solutions also exist, but they are not bound by the guide and represent the continuum of so-called radiating modes

It can be derived for the symmetric slab waveguide [7] that the number of guided modes $N$ is equal to

$N=2\left(\operatorname{ENTIRE}\left[d \omega\left(n_{1}{ }^{2}-n_{2}{ }^{2}\right)^{1 / 2} / c\right] / \pi+1\right)$

with $d$ as indicated in Fig 1 It can be seen that the number of guided modes is at least 2 and depends on frequency, slab thickness and refractive indices (If the optical indices at both sides of the slab are unequal, situations may occur in which no guided mode can be supported at all ) 
Note that in the limiting case $n_{1}=n_{2}$, the waveguide structure has disappeared Nevertheless, there are two 'guided' waves according to the formula The explanation is that there is one free wave (two-fold degenerate with respect to polarization), which proceeds exactly along the $z$-axis

We have confined ourselves to waves running in the $z$-direction However, a solution for this direction will be accompanied by equivalent solutions for any direction in the $y z$-plane If we consider a strip of finite width in the $y$-direction this degeneracy of waves running in the $y z$-plane is lifted In this case a standing wave character must be reached in both the $x$-and the $y$-directions (giving rise to two mode numbers)

The same can be expected for the case of the optical fibre If we consider a crrcular core surrounded by cladding of a lower index, we can expect guided wave solutions of Maxwell's equations again with an oscillatory structure inside the core, which fit to exponentially decaying parts in the cladding Analogous to the case of the slab, we can express the modes as follows

$E=E_{p, q}(x, y) \exp 1\left(\omega t-\beta_{p, q} z\right)$

Again the number of guided modes depends on the frequency, the core diameter and the indices of core and cladding

The ultimate structure of a mode is determined by the waveguide Characteristic for this structure is the spatial distribution of the electric/magnetic field vectors, which have both a magnitude and a direction So we have an amplitude or intensity distribution in a direction perpendicular to the waveguide axıs (the standıng wave structure), and a wavenumber characterizing the distribution of the running wave part along the waveguide axis The direction of the field vectors may give rise to specific polarization characteristics In our description frequency may also be considered as a characteristic of a fundamental light mode (the word mode now being used in a wider sense)

A cylindrical fibre supports at least two guided modes, which are degenerate because of the rotational symmetry Fibres that only support these two degenerate modes are called single-mode fibres. Although not selfevident, these modes can be chosen to have approximately planar or crrcular polarization just as in the case of free waves

We can characterize an arbitrary light wave supported by a waveguide as a distribution over the band of possible frequencies, and within a single frequency over the set of guided modes determined by the guide In principle, any superposition of modes can be prepared, each mode being characterized by its own mode structure and a coefficient $A$ that tells what the relative contribution of the particular mode is to the whole These coefficients are complex in general, carrying information about amplitude and phase

Figure 2 depicts this characterization schematically In the horizontal plane we have a frequency axis and a mode axis (for the sake of convenience we consider a single axis for the mode numbers/variables) A possible mode gives a point in the horizontal plane, for any frequency we have a discrete number of guided modes and a band of radiating modes The number of 


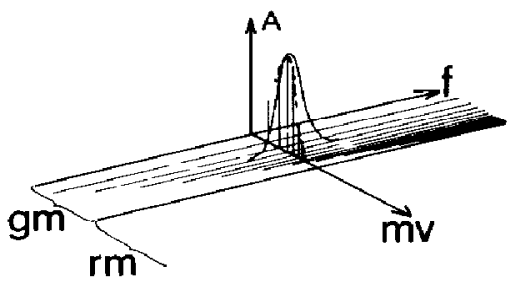

Fig 2 A light distribution can be characterized by the complex function $A(\omega, p)$ In the horizontal plane the set of possible $(\omega, p)$ values has a discrete structure for guided modes and forms a continuum for the radiating modes

guided modes depends on the frequency and the waveguide structure Along the third axis we denote the contribution (phase and amplitude) of each mode to the light wave as a whole In this way any light distribution can be characterized by the complex function $A(\omega, p)$ Elementary treatments of waveguide optics can be found in a great number of textbooks $[e g, 6-8]$

\section{Classification of optical waveguide sensors}

\section{Intrinsic sensors/extrinsic sensors}

As a starting point we use our definition of an optical waveguide sensor from the Introduction and classify according to the role of the waveguide in the sensor In doing so we have two categories, to which we now add a third

(1) Intrunsic sensors, in which the waveguide seems to play an essential role in the modulating mechanism

(2) Extrinsic sensors, in which the modulating principle is based on optics, but not on a waveguide property The waveguide plays an essential role nevertheless

(3) Same as 1 or 2 , but with an active role of the modulator

This classification, depicted schematically in Fig 3, approaches the one given by Kyuma et al [4] and gives a first division of the field, which is taken to be as wide as possible In the third category an additional optical branch is used to energize the modulator in order to start or maintain a process that is modulated by a measurand We will give some examples from this category in Section 4 In this Section we focus our attention on the passive intrinsic and extrinsic sensors

In intrinsic sensors the modulating effects have a distributed character As an example, consider a current sensor making use of the Faraday effect in a fibre that is colled around a current wire (see Fig. 4(a)) The same effect can be exploited by bulding such a sensor with specially prepared parts (see Fig 4(b)) and using fibres only as a means to transport light to and from the sensor area In the latter case we speak of an extrinsic sensor

The difference is that in the intrinsic sensor the light distribution in the modulator area is shaped by the waveguide and thus can best be described in terms of the mode spectrum defined by this waveguide In an extrinsic sensor the light distribution, in the region of interaction, can best be de- 
1. INTRINSIC

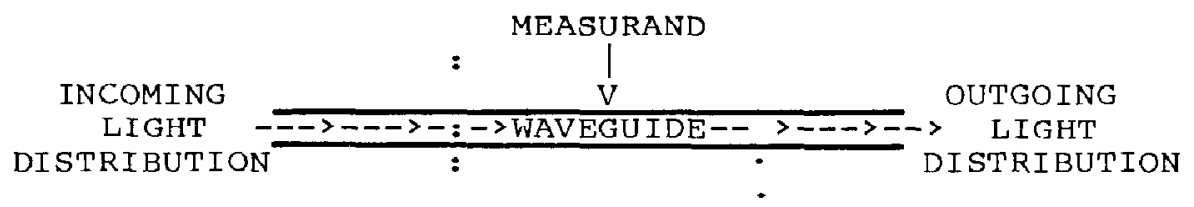

2. EXTRINSIC

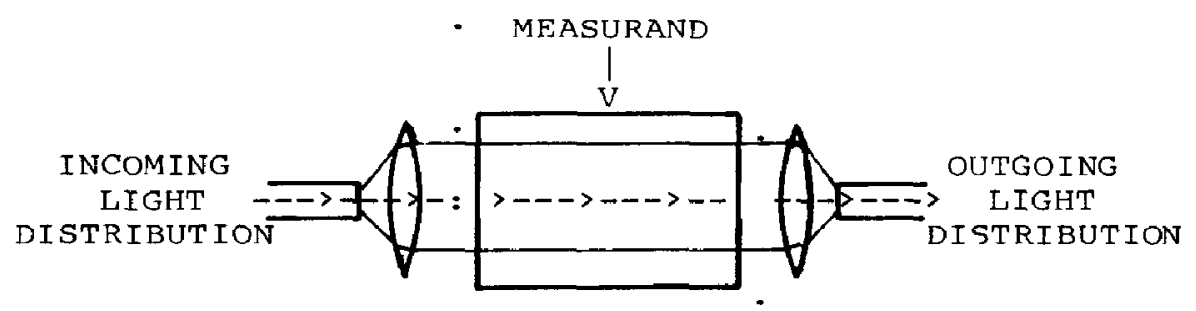

\section{ACTIVE}

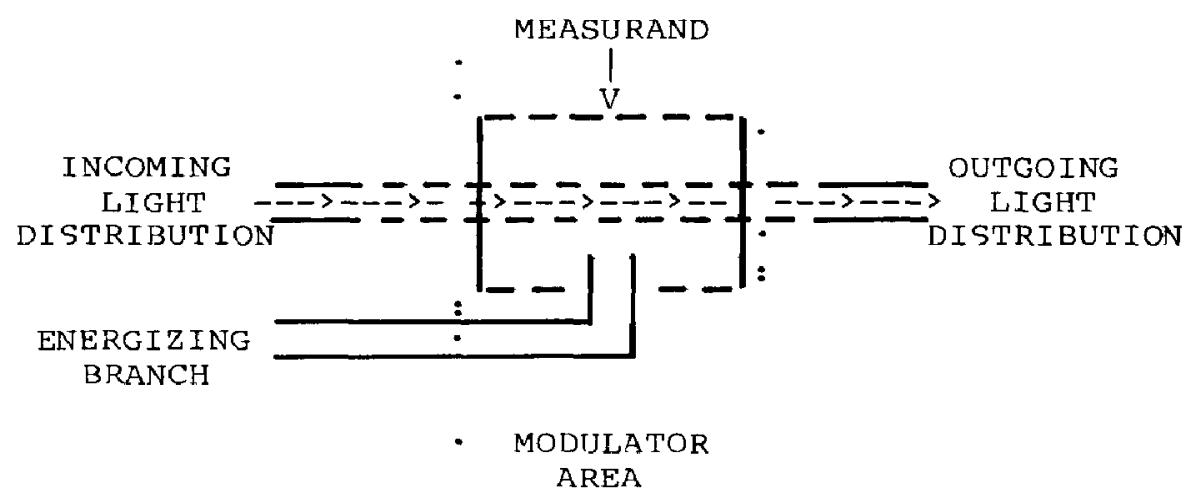

Fig 3 Classification of waveguide sensors

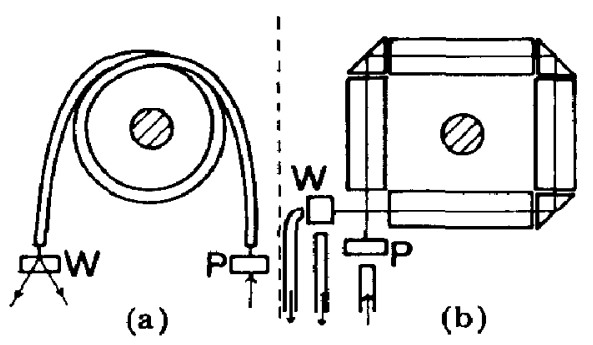

Fig 4 (a) Current sensor using a fibre colled around a current wire (intrinsic use of Faraday effect) (b) Current sensor with the light guided around the current wire using discrete components (extrinsic use of Faraday effect) In both cases the incoming light is polarized (P), while the outcoming light is analysed using a Wollaston prism (W) 
scribed with the help of free waves However, we can see a free wave distribution as an extreme form of 'guided light' (as shown in the preceding Section) The lens preceding the modulator area in Fig 3 serves as a means to guide the light into an axial direction, the light can then be considered as the 'guided free wave'

The conclusion is, that from the standpoint of the physical modulator mechanisms, the distinction intrinsic/extrinsic is not an elementary one and that the advantage of an intrinsic sensor mainly stems from the fact that well-shaped light can be made subject to a modulator mechanism in a very convenient way over a very long trajectory without expense of volume and costs

A further classification of (passive) optical waveguide sensors should be based on an analysis of the modulating process Roughly (and possibly not justifiably for all possible situations) this process consists of two steps, as can be denved from Fig 3 In the furst step the measurand alters the wavegunde, which is a geometrically specified distribution of suitable materials The material parameters of interest follow from the wave equation the dielectric tensor $\overline{\bar{E}}$ relating the electric displacement $\bar{D}$ to the electric field strength $\bar{E}$ $(\bar{D}=\overline{\bar{\epsilon}} \bar{E})$ and the magnetic permeability tensor $\overline{\bar{\mu}}$ relating the magnetic induction $\bar{B}$ to the magnetic field strength $\bar{H}(\bar{B}=\bar{\mu} \bar{H})$ Consequently a waveguide is defined by the functions $\bar{\epsilon}(\bar{r})$ and $\overline{\bar{\mu}}(\bar{r})$ with $r$ the coordinate vector

In the second step the modified waveguide modulates the light passing through it, which means that we must know how a guided light distribution should be characterized According to the preceding Section, this can be done by specifying the complex amplitudes $A(\omega, p)$ of the contributing modes and frequencies

We will treat the two steps of the process in the next subsections, starting with the latter

\section{Modulation of guided light}

In the most general case, the modulation of a light distribution can be obtained by measuring $A(\omega, p)$ at the fibre output and comparing this with $A(\omega, p)$ at the input $A(\omega, p)$ can in principle be measured by measuring the phase and the amplitude (or intensity) of the individual modes In order to separate the modes one needs spatial or temporal fultering, polarization filtering or whatever other technique can be used The actual measurement technique relies on the model underlying the sensor mechanism One knows beforehand what aspect of the hight distribution is related to the measurand This can be a mode amplitude or phase, a frequency shift, a mean value (of frequency or polarization in a certain direction) or an overall value (of intensity) or whatever The secret of designing optical waveguide sensors is to find a surtable combination of a modulating effect and a mode distribution that can easily be prepared and analysed

Let us consider a waveguide supporting only a single mode A change in phase and/or a change in amplitude is all that can occur In a perfect mono- 


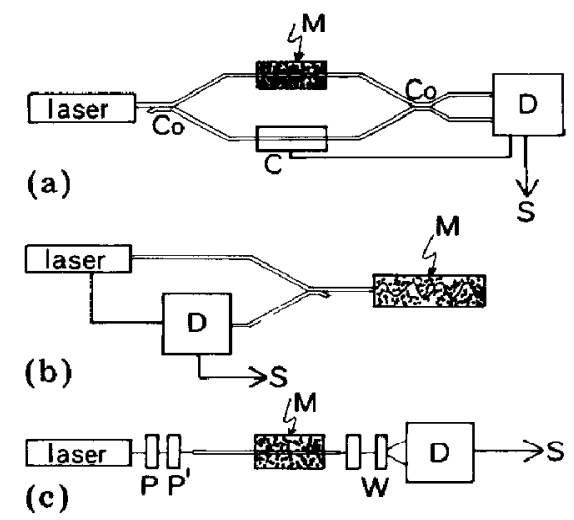

Fig 5 (a) Phase modulation can be measured in a two-branch interferometer with a resolution of $10^{-6} \cdot 10^{-8} \mathrm{rad}$ [2] One branch is under the influence of the measurand $M$, the other (C) can be controlled optically such that the phase sensitivity at the output is highest The system consists of a laser, a number of couplers (Co), a detector (D) and a control unit giving the signal (S) (b) A modulated Fabry-Perot filter can be kept in resonance by controlling the frequency of the laser The control unit gives the signal output (c) Polarimetric measurement (Resolution $10^{-3}-10^{-4}$ rad [2]) Laser light is linearly or curcularly polarized $\left(P, P^{\prime}\right)$ The modulated light is demodulated using a Wollaston prism (W) A Solell-Babinet compensator could be used for high sensitivity

chromatic guided wave the phase at the output is related to the phase at the input of the waveguide This relation is determined by the optical pathlength Any change of the latter leads to a phase shift at the output compared with the undisturbed situation This phase shift can be measured by means of interference with an undisturbed wave (Fig 5(a)), which is guided through a second branch to the detector [9] Phase modulation can also be detected in the time domain One way of detecting the delay effect is to lead the wave back and forth along the same trajectory with the help of partly reflecting murrors perpendicular to the axis of propagation. In this way one can obtain actually a modulated Fabry-Perot filter [9] (Fig 5(b))

A change in amplitude can be measured directly and interpreted if the amplitude at the input is known In practice, this means a second branch is needed as well The complete system may contain many parts and joints, which may be subject to loss of light power as a consequence of geometrical disturbances, temperature drift, etc So the ideal is a second branch with exactly the same trajectory as the furst, except for the modulator region

Changes in amplitude may be caused by absorption or emission, or by processes in which both appear in a correlated fashion ( $e g$, photoluminescence) We include such processes, which have the appearance of a frequency shift $A$ change in amplitude may also be caused by a scattering of power from the guided mode (we still consider the case of the waveguide supporting one single mode') into the radiating modes at a single frequency, since light in a radiating mode is considered as lost

Next we consider a waveguide supporting two modes Four quantities can now be changed two amplitudes and two phases With two modes in 
a single waveguide, the ideal of a reference branch is reached if the modulator mechanism has a differential effect on both modes. The secret is to find a modulator mechanism that distinguishes the modes, while disturbing effects do not

In a circular single-mode fibre the two modes differ in their polarization characteristics Depending on the problem under consideration, we can distinguish two plane-polarized modes or two curcularly-polarized modes Induced birefringence can lead to a differential effect on the phase of both modes This can be measured from the polarization characteristics of the light at the output (Note that one must have knowledge of these at the input side) Therefore, such sensors are called polarimetric sensors in the literature (Fig 5(c)) [9, 10] Differential effects on the amplitude lead to comparable effects (If a modulator mechanism works on the two modes simultaneously, the waveguide can be used in the same way as in the preceding example )

In wavegudes supporting more than two modes (in practice multimode fibres carry very many modes) the amount of information that can be acquired from a modulator mechanism with a differential effect increases with the number of modes On the other hand, the interference between modes as a consequence of disturbing effects and the difficulties in the analyses of the mode distribution at the output increase as well Therefore multumode waveguides are mostly used in sensors that modulate properties like the overall intensity, $l e$, properties that characterize the mode distribution as a whole and not in detal With respect to frequency characteristics, there seems to be no much distinction about the number of modes a waveguide can carry Typical waveguide properties have less to do with frequency than with the mode variables

In conclusion, we may state that optical waveguide sensors may be classified according to the type of modulation of the light distribution and the related measurement technique For individual modes the only quantities that can be modified are amplitude (intensity) and phase For a distribution of modes additional characteristics to be modulated can be identified, such as frequency, wavenumber and polarization

\section{Physical alteration of a waveguide}

A waveguide is specified by the tensor functions $\overline{\bar{\epsilon}}(r)$ and $\overline{\bar{\mu}}(r)$ The phenomena that are able to originate changes in these functions can be divided into two groups These can be derived from the fact that an element of volume $d V$ at location $\bar{r}$ with specified $\epsilon$ - and $\mu$-values can be subject to the following changes the element can be shifted in space, the element can be strained and $\epsilon$ or $\mu$ can change value as a function of frequency and mode variable

The geometrical changes are considered to belong to a first group, the other changes belong to a second group concerning material properties The distinction between these groups does not mean that physical processes 
actually act separately according to them A temperature change, for instance, may lead to a change of geometry and a change of $\epsilon$ at the same tume

Note that the quantities concerning geometry (position, strain) are state variables, while the quantities $\epsilon$ and $\mu$ are constitutional parameters

\section{Geometrical aspects}

The geometrical phenomena concern the mechanical domain, which in turn contains the kunetic and the potential (sub)domains a waveguide can be moved (giving it a velocity) or it can be strained This strain can be axial or radial, in a uniform or in a non-uniform fashion

The measurand might be one of the modifications mentioned here or one of the set of possible causes thereof. Direct causes are force fields Indirect causes originate from (generator type) coupling with other domains

piezoelectricity couples with the electric domain,

magnetostriction couples with the magnetic domain,

thermal expansion couples with the thermal domain,

'chemostriction' couples with the chemical domain

These couplings may act on the waveguide material itself or on a material structure supporting the wavegunde

The phenomena stated above obviously concern intrunsic effects Extrinsic effects concern geometrical aspects of parts inserted between fibre ends or at a tip. One can think of the movement or position of the fibre ends themselves, or of the movement or position of solid objects, fluids or gases in between These may give nse to effects on the intensity or the phase of the light used or on other characteristics ( $e g$, frequency if the Doppler effect is exploited)

\section{Material aspects}

The material aspects find their root in the phenomena that determine the values of the elements of the $\epsilon$ - and $\mu$-tensors These phenomena are related to the (pseudo)particle concept of matter, namely all sorts of electronic or lattice transitions and their respective dispersive side effects (Kramers-Kronig or Hilbert dispersion)

Contributing to $\epsilon$ are electronic vibrations, lattice vibrations, crystal field transitions, transitions in molecular orbitals, band transitions in metals or semiconductors, core transitions, etc

Contributing to $\mu$ are all sorts of magnetic resonances (ferri-, ferro-, antuferro-, etc ), optical transitions involving magnetic dipoles, etc

All elements of the tensors can in principle be expressed in electronic parameters when the mechanisms of contributing transitions are known Knowledge of these expressions, obtained as complex quantities, in turn allows for the introduction of a dependence on the parameters to be measured

A schematic representation of these dependences is given in Table 1 The phenomena in this Table denote only the relation between the observables and the physical domains Several fundamental mechanisms may be the 
TABLE 1

Examples of phenomena relating changes in the physical domains to changes in frequency $(\omega)$, intensity $(I)$ and polarization $(p)$ of light All examples give rise to changes in the tensor elements leading to variations in phase

$\begin{array}{ll}\text { Mechanical domain } & \text { Triboluminescence }(I, \omega) \\ & \text { Piezo-optic effects }(I) \\ & \text { Effects of local symmetry on transition }(I, p) \\ & \text { Raman-scattering }(I, \omega) \\ \text { Electric domain } & \text { Eathodoluminescence }(I, \omega) \\ & \text { Electroluminescence }(I, \omega) \\ & \text { Electrochromic effects }(I) \\ & \text { Pockels effect }(p) \\ & \text { Franz-Keldysh effects }(p) \\ & \text { Magnetic-absorptive effect }(p) \\ & \text { Magnetic birefringence }(I) \\ \text { Magnetic domain } & \text { Magnetic dispersion }(I) \\ & \text { Faraday rotation }(p) \\ & \text { Chemoluminescence }(I, \omega) \\ & \text { Franz-Keldysh effect }(I) \\ \text { Chemical domain } & \text { Electrochemical effects }(I) \\ & \text { Specific absorption }(I) \\ & \text { Thermoluminescence }(I) \\ & \text { Temperature dependence of effects } \\ \text { Thermal domain } & \text { Pg, quenching of luminescence }(I)) \\ & \text { Photoluminescence }(I, \omega) \\ & \text { Cathodoluminescence }(I, \omega) \\ \text { Radiative domain } & \text { X-ray luminescence }(I, \omega) \\ & \text { Photochromic effects }(I) \\ & \end{array}$

cause of these effects Here we will not dwell on correlating mechanisms and phenomena in general This would only be appropriate when specific materals have been chosen

\section{Examples}

With the help of the classification schemes of the preceding Section, we have selected a number of characteristic examples, which cover the entire field Diversity is pursued rather than completeness We have restricted ourselves to very concise descriptions together with some performance characteristics as given in the appropriate reference

Single-mode fibre, intrinsic

Temperature sensor [11] Single-mode fibre Influence on optical pathlength $(\phi=\beta L)$

The phase change per unit of length of fibre and of temperature $T$ is given by 
$\mathrm{d} \phi / L \mathrm{~d} T=\mathrm{d} \beta / \mathrm{d} T+\beta \mathrm{d} L / L \mathrm{~d} T$

There are two contributions, one concerning geometry (length), the other concerning geometry (radial) and materials (refractive indices).

According to ref 11 a sensitivity of $124 \mathrm{rad} / \mathrm{K} \mathrm{m}$ can be obtained in a two-fibre interferometer.

An alternative method is based on a differential influence on the two polarization modes A birefringence induced by a lateral stress is modulated according to

$\mathrm{d}(\Delta \phi) / L \mathrm{~d} T=\mathrm{d}(\Delta \beta) / \mathrm{d} T+\Delta \beta \mathrm{d} L / L \mathrm{~d} T$

Again we have two contributions, leading to a temperature sensitivity of 25 $\mathrm{rad} / \mathrm{K} \mathrm{m}$ in a polarimetric measurement. Although this sensitivity is much smaller than the one mentioned above $(50 \times)$, it has been shown that the cross sensitivity for pressure is very much smaller (7000X)

Figure 4 gives a general impression of a two-fibre and a polarimetric system

Acoustic sensor [3, 12] Single-mode fibre Influence on optical pathlength in low-frequency regime. Differential influence on polarization states in ultrasonic regime ( $40 \mathrm{MHz}$ )

If the acoustic wavelength is much larger than the dimensions of the sensor, the fibre experiences hydrostatic pressure vanations leading to phase shifts, which are detected with a two-fibre interferometer

If the acoustic wavelength is of the order of the fibre diameter, birefringence is induced and can be detected in a polanmetric measurement

Sensitivity is of the order of $10^{-4} \mathrm{rad} / \mathrm{Pa} \mathrm{m}$ in both cases

Fiber-optic gyroscope [13] Single-mode fibre Influence on optical path as a consequence of Sagnac effect (Fig 6)

According to theory, we have

$\Delta \phi=2 \pi^{2} D^{2} N \Omega / \lambda c$

with $N$ the number of turns of a coll with diameter $D$ rotating with rate $\Omega$ With $N=3000, D=10 \mathrm{~cm}$ and $\lambda=10^{-6} \mathrm{~m}$, a sensitivity of $2 \mathrm{rad} / \mathrm{rad} / \mathrm{s} \mathrm{can} \mathrm{be}$ obtained With a resolution of $10^{-7} \mathrm{rad}$, a rotation rate as small as $10^{-2} \% / \mathrm{h}$ can be measured'

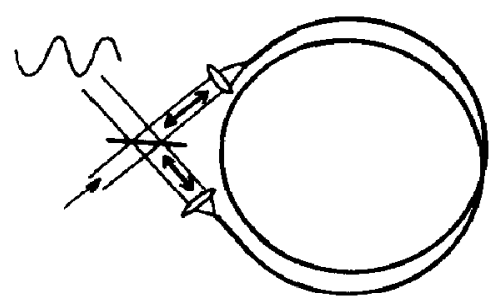

Fig 6 Fibre-optic gyroscope Light is fed into fibre coll clockwise and counter clockwise The phase difference at the output is a measure of the rotation rate 
Magnetic field sensor Single-mode fibre Magnetostrictive transduction of field to fibre strain

Using a fibre with a magnetostrictive jacket in a two-fibre interferometer, a sensitivity in the order of $10^{5} \mathrm{rad} / \mathrm{T} \mathrm{m}$ can be obtained in the frequency range $100 \mathrm{~Hz}-10 \mathrm{kHz}$ [3] With a resolution of $10^{-6} \mathrm{rad}$, an induction of $10^{-11} \mathrm{~T}$ can be detected with a fibre $1 \mathrm{~m}$ long

In a specific arrangement low-frequency $(0-20 \mathrm{~Hz})$ fields can be measured with a resolution of $2 \times 10^{-10} \mathrm{~T}$ per metre of fibre [14]

Rashleigh [15] coils a fibre around a magnetostrictive cylınder and makes use of the differential effect on polarization modes A sensitivity of $8 \times 10^{3} \mathrm{rad} / \mathrm{T} \mathrm{m}$ can be obtained

Electrical current sensor [16] Single-mode fibre Faraday effect using polarimetric detection

Using the Faraday effect a sensitivity of $2 V$ per turn, with $V$ the Verdet number $\left(45 \times 10^{-6} \mathrm{rad} / \mathrm{A}\right)$ can be obtained This leads to a sensitivity of $9 \times 10^{-6} \mathrm{rad} / \mathrm{A}$ turn With a resolution of $10^{-4} \mathrm{rad}$, a current of $01 \mathrm{~A}$ can be detected per fibre turn In practice, this method is used to detect large currents $(01$ - $1000 \mathrm{~A})$

Temperature sensor [17] Single-mode fibre Phase detection using a modulated Fabry-Perot filter

$26 \mathrm{~mm}$ of a single-mode fibre with polished end faces serves as a FabryPerot filter Temperature-induced shift of resonance is compensated by the injection current of a laser light source Range $35-55^{\circ} \mathrm{C}$, resolution $01{ }^{\circ} \mathrm{C}$

Figure 5 gives an impression of the detection scheme

Multımode fibre, intrinsic

Flowmeter [18] Multimode fibre Modulated mode-mode interference (fibredyne effect)

Fibre is mounted transversely in flow. As a consequence of the vortex shedding phenomenon, the fibre vibrates at a frequency that is approximately proportional to the flow. The phase shifts can be detected in the modal pattern at the fibre output Sensor was tested at flow rates of $03-3 \mathrm{~m} / \mathrm{s}$

Force, displacement sensor [19] Multimode fibre, graded index Mode coupling as a consequence of periodic ('resonant') microbending

Periodic bending with an adjusted periodicity (Fig 7(a)) couples the adjacent equidistant modes (In wavenumber space) As a consequence there is a flow of power from guided modes to radiation modes. With a number of bends of the order of 10 , a resolution of $10 \mathrm{~nm}$ can be reached The force resolution depends on the compliance of the system

Strain detector [20] Multimode fibre Microbending induced loss

Detection of strain in large structure ( $e$ g., off shore) Fibre is mounted in zig-zag fashion along pins or by bonding (Fig 7(b)) Strain induces sharp 


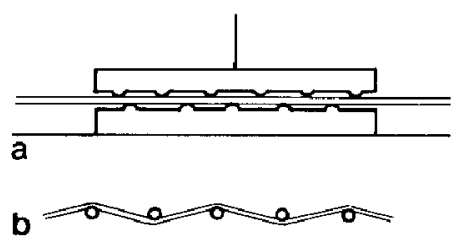

Fig 7 (a) Force-displacement sensor (b) Strain detector

bending and consequently a coupling of guided modes into radiating modes Mean strain over a length of $025 \mathrm{~m}$ can be measured with a resolution of 100 microstrain

Temperature sensor Multımode fibre, mode coupling as a consequence of modulated permittivity of cladding

At the end of a (sllica plastic) fibre the (slicone rubber) cladding is removed (Fig 8(a)) The fibre tip with a reflecting endface is placed in a

a
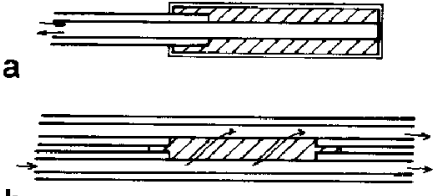

b

Fig 8 (a) Temperature sensor (b) Idem, with second fibre to collect radiated light

mineral oil with a strongly temperature dependent index of refraction in the trajectory 35 - 40 degrees centigrade The varying index difference between core and cladding changes guided modes to radıatıng modes Accuracy $01^{\circ}$ Biomedical application [21]

A second design uses two fibres with partly removed cladding (Fig 8(b)) A fluid with a temperature-dependent index of refraction (e $g$, motor oil) changes guided modes into radiation modes in one fibre while part of this light is caught in the second fibre [22]

Temperature sensor [23] Multımode fibre Temperature-dependent absorption by dissolved impurities

Fibre of alumino-sllicate glass with $5 \%$ Nd Temperature range 0 $800{ }^{\circ} \mathrm{C}$ Sensitivity $04 \mathrm{~dB} / \mathrm{K} \mathrm{m}$ Double frequency detection (one reference signal) Sensor consists of $2.5 \mathrm{~cm}$ of fibre colled in probe head

\section{Extrinsic sensors geometrical aspects}

Pressure sensor [24] Based on detection of small displacement of glass flat in the evanescent wave region of internal reflector

Input fibre has polıshed end surface at such an angle that all modes are internally reflected Light can be coupled into a glass flat if the latter is brought sufficiently close to fibre end (Fig. 9) Range of detection $(R)$ a few tenths of a micrometer Pressure range of device 0-3 bar Accuracy 5\% 


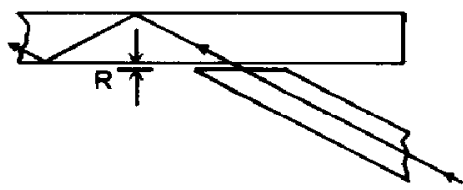

Fig 9 Pressure sensor

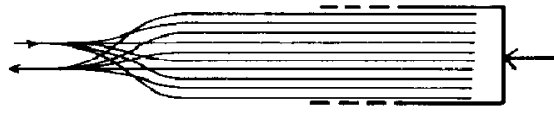

Fig 10 Displacement sensor

Displacement sensor $[25,26]$ Reflection from diaphragm

In one design [25], light from a modulated LED source is reflected from a diaphragm placed close to a fibre end The position of the diaphragm determines the fraction of light reflected into the fibre again A system to compensate for drift and aging is devised in this sensor, which works in a range of several hundreds of microns with a resolution of $5 \mu \mathrm{m}$ Application pressure sensing

A second design [26], developed for medical applications, uses fibre bundles to transport light to and from the membrane (Fig 10) In a range $(-13+40) \times 10^{-3} \mathrm{~Pa}$ a linear response within $1 \%$ is obtained

Positıon sensor, temperature sensor [27] Coupling of light between fibre ends

Light from an incoming fibre is coupled into a parr of collecting fibres via a ball lens This lens can be moved laterally and modulates the intensity ratio of light received by the collecting fibres (Fig 11(a)) Sensitivity of the order of $1 \mathrm{~dB} / \mu \mathrm{m}$. Application flow measurement

Light from an incoming fibre is reflected from a mirror with a half ball lens mounted on it If the murror is tylted, the intensity ratio of the light collected by the recelving fibres is modulated (Fig 11(b)) The tilt is introduced by the thermobimetallic mounting of the murror. Sensitivity $025 \mathrm{~dB} /$ K. Linear range $20-90{ }^{\circ} \mathrm{C}$
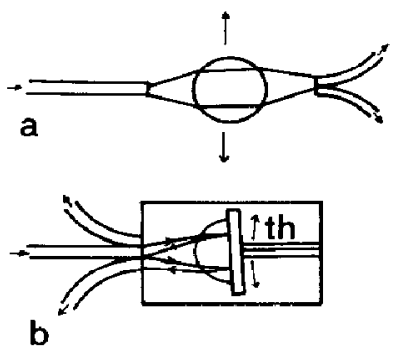

Fig 11 (a) Position of ball lens couples light between fibre ends (b) Temperature sensor with thermobimetal (th)

Accelerometer [28] Reflection from spring loaded murror

Cantlever beam reflector is mounted at fibre end (Fig 12) Acceleration tults the reflector and modulates the intensity of light reflected into the fibre again Part of incident light is absorbed and re-emitted as a consequence 


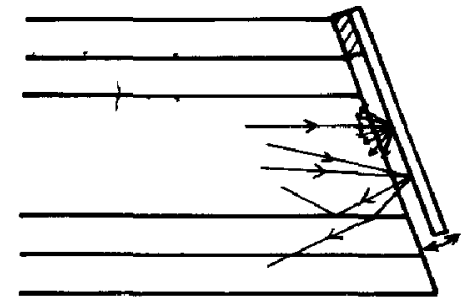

Fig 12 Accelerometer

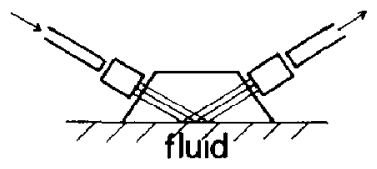

Fig 13 Fluid detector

of photoluminescence In this way a reference is created that is independent of the tilt of the reflector

Extrinsic sensors material aspects

Leak detector [29] Internal reflection on a prism face is modulated by the presence of liquid at the surface (Fig 13)

The choice of the angle of incidence is important to discriminate between fluds, $e g$, oll and water Application long distance leak detection of pipeline or onl-filled cable An oll-covered surface leads to a $10 \mathrm{~dB}$ decrease in reflected power

Electric current sensor [30] Faraday effect in single crystal

Use is made of a $\mathrm{Bl}_{12} \mathrm{GeO}_{20}$ single crystal Verdet constant $6 \times 10^{-5}$ $\mathrm{rad} / \mathrm{A}$ Resolution is about $1 \mathrm{~A} / \mathrm{m}$ (magnetic field) Temperature dependence within range of measurement $\left(-25+85{ }^{\circ} \mathrm{C}\right)$ is about $2 \%$ Same material can be used for voltage measurement Application in electric power systems

Pressure sensor Photoelastic effect

Using glass, a dynamic range of $120 \mathrm{~dB}$ is obtained Minimum detectable pressure $21 \mathrm{~Pa}$ Hysteresis, $08 \%$ of full scale $(6.9 \mathrm{MPa})$ [31]

Using specific material (a polycarbonate) the effect is exploited in underwater hydrophony [32]

Gas detector [33] Absorption of specific spectral line

Detection of absorption spectrum around $16 \mu \mathrm{m}$ in order to detect the presence of methane (Fig. 14) A second frequency outside the absorption is used as a reference $700 \mathrm{ppm}$ of gas in arr can be detected remotely (2km of fibre)

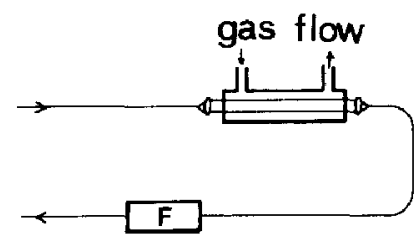

Fig 14 Gas detector Output branch contains filter (F) to separate frequencies 
Temperature sensor [34] Temperature-dependent photoluminescence

Light from an LED is absorbed by a photoluminescent semiconductor structure at a fibre tip. The re-emitted spectrum shifts to higher wavelength as the temperature increases (Fig 15) The ratio of the signals from two

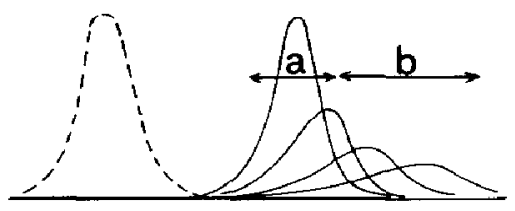

Fig 15 Temperature sensor Dashed curve incident light Solid curve re-emitted light at different temperatures Spectral range of two detectors is given by $a$ and $b$

detectors with different spectral sensitivities is a measure of the temperature Within the range $0-200^{\circ} \mathrm{C}$ an accuracy of $1^{\circ} \mathrm{C}$ and a resolution of $01{ }^{\circ} \mathrm{C}$ have been obtained

Molsture detector [35]

Cobalt(II) chloride is cast as films on the surface of $600 \mu \mathrm{m}$ optical fibres The absorption of these films depends on the presence of moisture because of the formation of hydrates with molecules of bound water Sensors for a humidity range $40-80 \% \mathrm{r} h$ have been obtained

Integrated sensors

Sensor for electromagnetic field [36, 37] Electro-optic effect

One design [36] uses a single-mode planar Mach-Zehnder interferometer using $\mathrm{T}_{1} \mathrm{LiNbO}_{3}$ ( F'ig 16) As a consequence of asymmetry, there is a
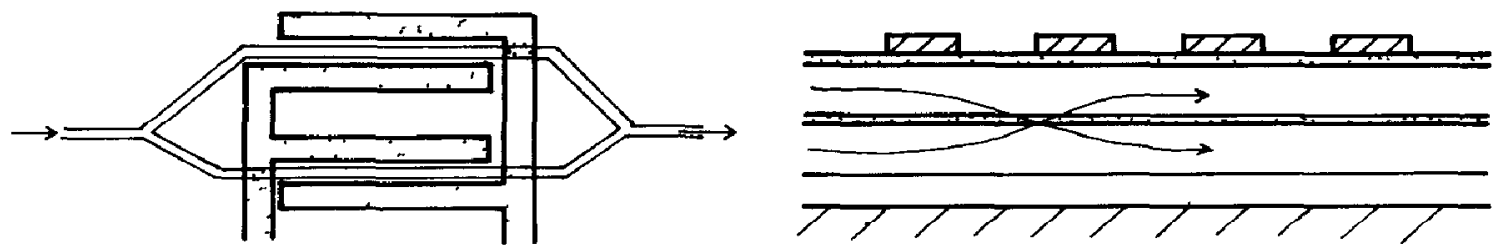

Fig 16 Electric field, voltage detector

Fig 17 Electric field, voltage detector (second design)

phase difference between the arms This phase difference is influenced by a voltage as a consequence of the electro-optic effect Linear operation (dynamic range $49 \mathrm{~dB}$ ) has been demonstrated over the range $0-300 \mathrm{MHz}$

A second design [37] uses a sandwich of planar waveguides, one of which is electro-optically sensitive (Fig 17) The voltage is applied to a periodic structure The period is chosen such that mode coupling is induced between a mode that is guided mainly through the upper channel and a mode that is guided mainly through the lower channel Complete coupling is expected for a voltage of the order of $10 \mathrm{~V}$ 
Pressure sensor [38] Elasto-optic effect

The sensor consists of an integrated version of a Michelson interferometer Pressure can be applied in one branch, which influences the optical path via the elasto-optic effect

Chemical sensor [39, 40] Coupling of modes Dispersive properties of a wavegunde are modulated by the presence of chemical compounds outside the waveguide. The modulation is accomplished by a coupling of guided modes with surface plasmons of a thin silver layer that is positioned between the wavegurde and the compound

\section{Active sensors}

General principle ( $\mathrm{F}$ ig 3 ) Light energy is fed to the transducer and converted into the desired form, say electric energy The signal acquired by the transducer is converted with an optical waveguide sensor A demonstration of the principle is given by Jones and Philp [41], in which a vibrating wre sensor is included in an optic transmission system

If transduction to the electrical domain takes place, all 'classical' transducers are within reach in principle

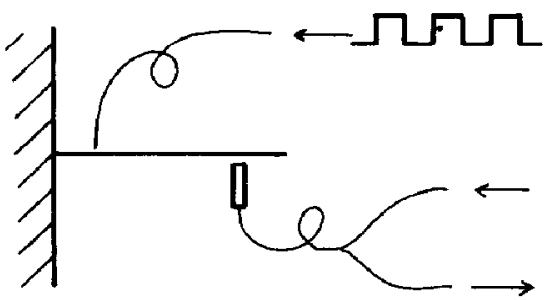

Fig 18 Opto-mechanical resonator

Opto-mechanical transduction Conversion to the electric domain is not necessary. A vibrating membrane sensor can be activated directly [42] by thermal expansion, while the vibration is sensed by optical means [43]

An alternative design uses a vibrating bar [44] (Fig 18)

All-optic telephone This has been described as well [45]

\section{Eplogue}

During the last few decades a new field of research concerning optical sensors has emerged, thanks to the development of the optical fibre This field is characterized by a great diversity of physical principles which, although not new in themselves, can be explored and made profitable

The specific advantages of optical measurement can be utılized at places which have been inaccessible for such techniques up to now In fact, this is a consequence of the fact that a fibre can be seen as an extended light 
source of very small dimension and very well defined light On the other hand, a fibre can be seen as an extended detector that can collect light in a well determuned way, again without expense of volume.

Apart from these advantages, which are applied in extrinsic sensors, the intrinsic type of sensor makes use of the fact that the sensitivity of certain physical effects is proportional to the length of fibre used. Incredibly sensitive devices can in principle be made, by coiling thousands of meters of fibre in a small volume

Active devices, powered via an optical fibre and sending the acquired signal in return along an optical path, are rare at the moment Nevertheless the concept is considered to be very fruitful

Integrated optical sensors are considered to be very promising In spite of the problems of integrating such parts into an optical network, integrated optics can fully exploit the flexibility of planar technology with respect to the use of materials, forms and production methods

The diversity of sensor principles makes it difficult to classify them in an orderly scheme Nevertheless, it is necessary to develop classification schemes in order to get some grip on the matter and we have put some emphasis on this On the other hand, order tends to exclude the unexpected, so any such scheme must be seen as an expedient rather than as a prescription to be followed strictly.

\section{References}

1 R P Main, Fibre optic sensors - future light, Sensor Review, (July) (1985) 133 - 139

2 B Culshaw, Optical Fibre Sensing and Signal Processing, Peter Peregrinus Ltd, London, 1984

3 T G Giallorenz1, J A Bucaro, A Dandridge, G H Sigel Jr, J H Cole, S C Rashleigh and $J$ H Priest, Optical fibre sensor technology, IEEE $J$ Quantum Electron, $Q E-18$ (1982) 626-665

$4 \mathrm{~K}$ Kyuma, S Tal and $M$ Nunoshita, Development of fibre optic sensing systems - A review, Optics and Lasers in Engineering, 3 (1982) 155 - 182

$5 \mathrm{~S}$ Yao and $\mathrm{C} \mathrm{K}$ Asawa, Fibre optical intensity sensors, IEEE $J$ Selected Areas Commun, SAC-1 (1983) $562-575$

6 M K Barnoskı (ed), Introduction to Integrated Optıcs, Plenum Press, New York, 1973

7 D Marcuse, Theory of Dielectric Optical Waveguides, Academic Press, New York, 1974

8 N S Kapany and J J Burke, Optıcal Waveguıdes, Academic Press, New York, 1972

$9 \mathrm{R}$ Kist and $\mathrm{R}$ Th Kersten, Fiber and integrated monomode sensors A review, Laser Optoelektron, 16 (1) (1984) $17-30$

$10 \mathrm{~S} C$ Rashleigh, Origins and control of polarization effects in single mode fibers, $J$ Lightwave Technol, LT-1 (2) (1983) 312 - 331

11 W Eickhoff, Temperature sensing by mode-mode interference in birefringent optical fibers, Opt Lett, 6 (4) (1981) $204-206$

$12 \mathrm{R} P$ dePaula, $J \mathrm{H}$ Cole and $\mathrm{J} A$ Bucaro, Broad-band ultrasonic sensor based on induced optical phase shifts in single-mode fibers, $J$ Lightwave Technol, LT-1 (2) (1983) $390-393$

$13 \mathrm{R} A$ Bergh, $H$ C Lefevre and $H$ J Shaw, An overview of fibre-optic gyroscopes, $J$ Lightwave Technol, LT-2 (2) (1984) 91 - 107 
14 A D Kersey, D A Jackson and M Corke, Single-mode fibre-optic magnetometer with DC bias field stabilization, $J$ Lightwave Technol, $L T-3$ (4) (1985) 836 - 840

$15 \mathrm{~S} C$ Rashleigh, Magnetic field sensing with a single-mode fibre, Opt Lett, 6 (1) (1981) $19-21$

$16 \mathrm{~S} \mathrm{C}$ Rashleigh and $\mathrm{R}$ Ulrıch, Magneto-optic current sensing with birefringent fibers, Appl Phys Lett, 34 (11) (1979) $768-770$

$17 \mathrm{R}$ Kist, S Drope and $\mathrm{H}$ Wolfelschnelder, Fiber-Fabry-Perot (FFP) thermometer for medical applications, Proc 2nd Int Conf on Opt Fibre Sensors, Stuttgart, September 1984, pp $165-170$

$18 \mathrm{~J} \mathrm{H}$ Lyle and C W Pitt, Vortex shedding fluid flow meter using optical fibre sensor, Electron Lett, 17 (1981) 244

$19 \mathrm{~W} H \mathrm{G}$ Horsthuis and $J \mathrm{H} J$ Fluitman, The development of fibre optic microbend sensors, Sensors and Actuators, 3 (1982/1983) $99-110$

20 A J A Bruinsma, P van Zuylen, C W Lamberts and A J T de Krijger, Fibre-optic strain measurement for structural integrity monitoring, Proc 2nd Conf on Opt Fibre Sensors, Stuttgart, September 1984, pp $399-402$

21 A $M$ Scheggı, M Brencı, G Confortı, R Falcıal and G P Pretı, Optıcal fibre thermometer for medical use, Proc Ist Int Conf on Upt Fibre Sensors, London, April 1983, pp $13-16$

22 S Ramakrishnan and $R$ Th Kersten, A multipurpose cross talk sensor using multimode optical fibres, Proc 2nd Int Conf on Opt Fibre Sensors, Stuttgart, September 1984, pp $105-110$

23 E Snitzer, W W Morey and W H Glenn, Fibre optic rare earth temperature sensors, Proc 1st Int Conf on Opt Fibre Sensors, London, Aprll 1983, pp $79-82$

24 K T V Grattan, A W Palmer and D P S Samı, Fruștrated-total-internal-reflectıon fibre-optic pressure sensor, $J$ Lightwave Technol, LT-3 (5) (1985) 1130 - 1134

25 D E N Davies, J Chaimowics, G Economou and J Foley, Displacement sensor using a compensated fibre link, Proc 2nd Int Conf on Fibre Sensors, Stuttgart, September 1984,pp $387-390$

26 T Hansen, A fibreoptic micro-tip pressure transducer for medical applications, Sensors and Acuators, 4 (1983) 545 - 554

27 H Dotsch, G Martens and W Meyer, Fibre optics components for industrial control, Proc 1st Int Conf on Opt Flbre Sensors, London, Aprl 1983, pp 67-71

28 L Jonsson and B Hok, Multımode fibre-optıc accelerometers, Proc 2nd Int Conf on Fiber Sensors, Stuttgart, Septem ber 1984, pp $191-194$

29 M Nagal, $M$ Shımızu and $N$ Ohgi, Sensitıve liquid sensor for long distance leak detection, Proc 2nd Int Conf on Opt Fabre Sensors, Stuttgart, September 1984, pp $207 \cdot 210$

$30 \mathrm{~K}$ Kyuma, S Ta1, M Nunoshita, N Mikamı and $\mathrm{Y}$ Ida, Fibre-optic current and voltage sensors using a $\mathrm{Bi}_{12} \mathrm{GeO}_{20}$ single crystal, $J$ Lightwave Technol, LT-1 (1) (1983) $93-97$

31 W B Spllman Jr and D C McMahon, Multımode fibre optic sensors, Proc 1st Int Conf on Opt Fibre Sensors, London, April 1983, pp $160-163$

$32 \mathrm{D} H$ McMahon, $\mathrm{R}$ A Soref and L E Sheppard, Sensitive fieldable photoelastic fibre-optic hydrophine, $J$ Lightwave Technol, $L T$-2 (4) (1984) 469 - 478

$33 \mathrm{~K}$ Chan, $\mathrm{H}$ Ito and $\mathrm{H}$ Inaba, An optical-fibre-based gas sensor for remote absorption measurement of low-level $\mathrm{CH}_{4}$ gas in the near-infrared region, $J$ Lightwave Technol $L T-2$ (3) (1984) $234-238$

$34 \mathrm{C}$ Ovren, $M$ Adolfsson and B Hok, Fibre-optic systems for temperature and vibration measurements in industrial applications, Proc Int Conf on Opt Technol in Process Control, The Hague, June 1983, 67-82

35 A P Russel and $K$ S Fletcher, Optical sensor for the determination of moisture, Anal Chimica Acta, 170 (1985) $209-216$

36 C H Bulmer, R P Moller and W K Burns, Linear Mach-Zehnder Interferometers in $\mathrm{LiNbO}_{3}$ for electromagnetic field sensing, Proc 2 nd Eur Conf on Integr Opt, Florence, October 1983, pp $140-143$ 
37 W $\quad H \quad G$ Horsthuis and $R$ Pannekoek, Electrooptic modulators in multilayered zincoxide waveguides, Springer Sertes in Optical Science, Vol 48, Integrated Optics, Springer Verlag, Berlin, 1985, pp $152-157$

38 M Izutsu, A Enokihara, N Mekada and T Sueta, Optıcal-waveguide pressure sensor, Proc 2nd Eur Conf on Integr Opt, Florence, October 1983, pp 144-147

39 C Nylander, $B$ Liedberg and $T$ Lind, Gas detection by surface plasmon resonance, Sensors and Actuators, 3 (1982) 79 - 88

40 H Kreuwel, Planar waveguide sensors based on mode-coupling for the measurement of electrical and chemical parameters, Collection of papers cancerning new developments on solld-state sensors, Techn Unıv Delft, 1985

41 B E Jones and G S Philp, A vibrating wre sensor with optical fibre links for force measurement, Fur Conf Sensors and therr Appl, Manchester, 198.3, Abstr 051

42 E Dieulesaint, D Royer and C Bonnefoy, Mechanical excitation of a membrane by an optical beam, Proc 1981 20th IEEE Uttrasontc Symposium, Chicago, October $1981, \mathrm{p} 802$

$43 \mathrm{~S}$ Venkatesh and B Culshaw, Optically activated vibrations in a micromachined sllica structure, Electron Lett, 21 (1985) 315 - 317

44 B Hok and S J Gerritsen, Multimode fibre optic sensors with frequency output, to be published in Int Opt Sensors

45 S R Adhav, B G Grossman, L M Ralston and R K Morse, The non-metallic, nonelectronic, optical telephone, Comm Int, 10 (1983) $97-101$

\section{Biographies}

Jan $H d$ Fluitman was born in Beverw1]k, The Netherlands, in 1938 He received the $\mathrm{M} \mathrm{Sc}$ and $\mathrm{Ph} \mathrm{D}$ degrees in physics from the University of Amsterdam in 1966 and 1970 respectively. Since 1970 he has been with the Department of Electrical Engineering, Twente University of Technology, Enschede, The Netherlands, currently as a Professor of Transduction Science

Theo $J$ A Popma was born in Heerenveen, The Netherlands, in 1941 He recelved the $\mathrm{M} \mathrm{Sc}$ and $\mathrm{Ph} \mathrm{D}$ degrees from the University of Groningen in 1966 and 1970 respectively During 1970 and 1971 he was with the IBM Thomas $J$ Watson Research Center in Yorktown Heights (N Y)

In 1971 he joined the Philips Research Laboratories in Eindhoven where he was employed untll 1984 In 1983 he was appointed Professor of Materials Science in the Departments of Applied Physics and Electrical Engineering of the Twente University of Technology at Enschede, The Netherlands 